\title{
Krise ohne Konflikt? Zur Wahrnehmung der Wirtschaftskrise aus Sicht von Betroffenen
}

\author{
Richard Detje/Wolfgang Menz \\ Sarah Nies/Dieter Sauer
}

\begin{abstract}
Auf den ersten Blick ist die „Große Krise“ seltsam friktionslos verlaufen: eine Krise, die Systemgrenzen des Finanzmarktkapitalismus aufgezeigt, aber wenig soziales Protestpotenzial mobilisiert hat. Aber stimmen die Diagnosen einer „Krise ohne Konflikt“ oder einer „Krise ohne Krisenbewusstsein“ auch bei näherem Hinsehen? Eine explorative Studie zur Wahrnehmung der Wirtschaftskrise zeigt: Hinter der vergleichsweise geräuschlosen Krisenverarbeitung schimmern verallgemeinerte Ohnmachtserfahrungen auf, es wird aber auch erhebliches, allerdings recht diffuses Protestpotenzial sichtbar.
\end{abstract}

\section{Einleitung: Krise ohne Konflikt?}

„Eine Gleichzeitigkeit von Krise und Konflikt fehlt heute vollkommen", konstatierte Claus Offe auf dem Höhepunkt der Krise 2009, als die Produktion in einigen Industriebereichen um ein Drittel und mehr eingebrochen war (Offe 2010) - ein Einbruch, der nur mit der Großen Depression der 1930er Jahre zu vergleichen ist (Eichengreen/O'Rourke 2010). Eine Krise zumal, von der niemand verlässlich sagen kann, ob sie überwunden ist oder nur in anderen Formen - als Verschuldungs- und Währungskrise und damit erneut als Finanzmarktkrise, die auf die Realökonomie zurückschlägt - fortexistiert. Derzeit spricht vieles für Letzteres. Offes Diagnose stimmt offensichtlich nicht für weite Teile Europas. In Griechenland, Italien, Spanien und Portugal kam es mehrfach zu Generalstreiks und anderen Protestaktionen, in Frankreich wurden Unternehmer in Geiselhaft genommen („boss napping“), in Großbritannien, Irland und Portugal stürzten Regierungen. In Deutschland hingegen scheint das ökonomische Jahrhundertereignis vergleichsweise geräuschlos über die Bühne zu gehen. „Weiter so“ wie vor der Krise scheint die Maxime der wirtschaftlichen und politischen Eliten zu sein - und niemand da, sie zu stoppen? Eine Krise, in der die "systemischen Risiken“ des Finanzmarktkapitalismus aufbrachen
- aber ohne gesellschaftliche Lernprozesse, mit ruhiggestelltem sozialem Konflikt?

Verschiedene Erklärungen werden für den konfliktarmen Krisenverlauf angeführt:

(1) In erster Linie habe das „deutsche Beschäftigungswunder“ („german miracle“, Economist, 11. Mai 2010) für einen glimpflichen Krisenverlauf gesorgt. Die Abwesenheit von Konflikten erklärt sich in dieser Perspektive schlicht aus der Abwesenheit ernsthaft spürbarer Krisenfolgen. Genauer hingeschaut, zeige sich hier die Möglichkeit der Reaktivierung einer korporatistischen Verhandlungsökonomie, die einstmals zum Erfolg des „Modell Deutschland “ beigetragen hatte und in der Krise eine größere Outputlegitimation unter Beweis gestellt habe als die Reaktivierung des industriellen Konflikts.

(2) Wilhelm Heitmeyer argumentiert mit einem Stadienmodell, das unterschiedliche Betroffenheiten in der Krise skizziert. Im ersten Stadium der Finanzmarktkrise werde die Bedrohung als rein virtuell, nicht handlungsleitend erfahren; Stadium 2 der Wirtschaftskrise erreiche zwar die Arbeits- und Lebenswelten, fragmentiere aber zugleich; im Stadium 3 der Fiskalkrise nähmen die Wohlstandskonflikte, zugleich aber auch die Desintegrations- und sozialen Abwertungsprozesse zu; daraus resultiere in Stadium 4 eine Gesellschafts- und Staatskrise, die ohne ,institutionelle Lernprozesse“ schließlich in einen ,autoritären Kapitalismus“ münden könne (Heitmey- er 2010a, S. 18ff.). Statt zu höherem Konfliktpotenzial im gesellschaftlichen Unten führe die Krise zu einer „entsicherten wie entkultivierten Bürgerlichkeit" der Eliten (Heitmeyer 2010b, S. 15; Heitmeyer 2010c).

(3) Ein weiterer Erklärungsansatz konstatiert, dass vor allem in den besonders krisenbetroffenen unteren sozialen Schichten sich Fatalismus ausgebreitet habe (Köcher 2009, S. 15). Zwar lasse die Prekarisierung der sozialen Verhältnisse die Krisenfolgen

\footnotetext{
Richard Detje ist Mitglied der Wissenschaftlichen Vereinigung für Kapitalismusanalyse und Gesellschaftspolitik (WISSENTransfer) und Redakteur der Zeitschrift Sozialismus. e-mail: buero@wissentransfer.info Wolfgang Menz, Dr., ist Sozialforscher am Institut für Sozialwissenschaftliche Forschung e.V. (ISF) München. Arbeitsschwerpunkte: Leistungs- und Gesundheitspolitik, Arbeit und Subjekt. e-mail:wolfgang.menz@isf-muenchen.de Sarah Nies ist Promotionsstipendiatin der Hans-Böckler-Stiftung und Sozialforscherin am ISF München. Arbeitsschwerpunkte: Leistungspolitik, Interessenpolitik, Arbeit und Subjekt. e-mail: sarah.nies@isf-muenchen.de Dieter Sauer, Prof. Dr., ist Sozialforscher am ISF München und Honorarprofessor für Soziologie an der Friedrich-Schiller-Universität Jena. Arbeitsschwerpunkte: Reorganisation von Unternehmen, Vermarktlichung und Subjektivierung von Arbeit. e-mail: dieter.sauer@isf-muenchen.de
} 
besonders spürbar werden, verhindere aber zugleich einen offensiven Umgang mit ihr, sodass keine neuen Kollektividentitäten und alternative Bewältigungsstrategien entstünden. Die Gesellschaft verharre in einem Zustand der Spaltung zwischen „Statusfatalismus" unten und ausgrenzendem Pochen auf die Macht des Stärkeren aufseiten der Bessergestellten.

(4) Das Jenaer Forscherteam um Klaus Dörre ist „auf eine Grundstruktur des Arbeitsbewusstseins gestoßen, die sich in der Formel ,guter Betrieb - schlechte Gesellschaft' zusammenfassen lässt" (Dörre et al. 2010, S. 1). Während in der Gesellschaft soziale Ungerechtigkeitserfahrungen stark zugenommen hätten, erweise sich das Unternehmen in der Krise als „Garant sozialer Stabilität“". Wenn aber zwischen ,identitätsstiftendem Betriebs- und kritischem Gesellschaftsbewusstsein ... subjektiv kein Zusammenhang“" (ebd., S. 2) besteht, erwachse aus Angst vor sozialem Abstieg ähnlich wie in der Diagnose Heitmeyers - die Gefahr autoritärer Herrschaft.

Die genannten Thesen bieten bislang allerdings trotz interessanter Interpretationsansätze nur fragmentarische Einsichten in das Krisenerleben von Beschäftigten. Die betriebliche Ebene - und gerade hier dürften ja relevante Krisenerfahrungen ihren Ort haben - bleibt zumeist außer Acht. ${ }^{1}$ Die Wahrnehmung der Krise, Einblicke in ihre Ursachen, die Bewertung der Folgen, die Klärung von Interessenpositionen und Handlungsoptionen erfolgt aber vor allem im arbeitsgesellschaftlichen Nahbereich. Deshalb haben wir Erhebungen gleichsam im Zentrum der Krise in Deutschland durchgeführt, nämlich mit Betroffenen aus der Metall- und Elektroindustrie (u. a. Automobilindustrie) sowie aus der textilen $\mathrm{Au}$ tomobilzulieferindustrie. Im Frühsommer 2010 haben wir betriebliche Vertrauensleute und - wo es sie nicht gab - Betriebsräte in ausführlichen Interviews und Gruppendiskussionen befragt, also die unterste Ebene der betrieblichen Interessenvertretung, die in gewisser Weise zwischen Beschäftigtenperspektive einerseits und Expertenperspektive andererseits vermitteln kann. Die Ergebnisse aus unserem explorativen Forschungsprojekt (vgl. ausführlich Detje et al. 2011; siehe auch Menz et al. 2011) $)^{2}$ können das bisherige unvollständige Bild des betrieblichen und gesellschaftlichen Krisenbewusstseins ergänzen und erweitern und zugleich neue Antworten auf die Frage nach den (ausgebliebenen?) Konflikten und Widerstandsformen geben.

Nachfolgend umreißen wir zunächst das Spektrum der Krisenwahrnehmungen und des Krisenerlebens (Abschnitt 2). Anschließend stehen die betrieblichen Maßnahmen der Krisenbewältigung im Mittelpunkt: Wie werden sie bewertet und eingeschätzt? Was ereignete sich unter der Oberfläche des „deutschen Beschäftigungswunders" (Abschnitt 3)? Im Abschnitt 4 blicken wir über die betriebliche Ebene hinaus auf die gesellschaftlichen Perspektiven von Protest. Trifft die verbreitete Diagnose einer weitgehend pazifizierten Krisenbewältigung in Deutschland zu? Abschließend resümieren wir unsere empirischen Ergebnisse vor dem Hintergrund der eingangs skizzieren Thesen (Abschnitt 5).

\section{2 \\ Vielfalt der Krisenwahr- nehmungen - Intranspa- renz der Ökonomie}

Was und wann Krise ist, ist nicht selbstverständlich. Auch dort, wo die „neue Weltwirtschaftskrise“ (Krugman 2009) die tiefsten Spuren hinterlassen hat, ist das Bild ausgesprochen differenziert. Stellvertretend für die Vielfalt der Krisenwahrnehmungen skizzieren wir drei Typen: ${ }^{3}$

(a) Im ersten Wahrnehmungstypus der "Krise als herber Schlag" wird die Krise als deutlicher Einschnitt - sowohl in ihren Folgen für den Betrieb wie auch für den einzelnen Beschäftigten - wahrgenommen: „... vor drei Jahren hattest du ganz andere Perspektiven, ganz andere Vorstellungen. [...] Und dann hast du plötzlich... so eine Krise. ... da bist du wieder eigentlich zurückgeschossen worden. Da warst du wieder ganz unten ... das war schon ein herber Schlag. " 4

Der „Schlag“ hatte durchaus die Qualität eines Schockerlebnisses - so etwas hatte man vorher noch nie erlebt. Zugleich ist für diese Beschäftigten aber auch typisch, dass die Krise wiederum ein definierbares Ende hat. Krise erscheint eher als Übergangssituation, oder - wie ein Befragter formuliert - „wie eine Grippe“, die einen niederhaut, von der man sich nach gewisser Zeit aber wieder erholt. (b) Häufiger allerdings wird die „Krise als permanenter Prozess“ erlebt. Es ist nicht so sehr eine konkrete Krisensyndromatik zu einem bestimmten Zeitpunkt, sondern ein längerfristiger Prozess der Krisenhaftigkeit, der für diese Wahrnehmung kennzeichnend ist. Die große Wirtschaftskrise ist zwar durchaus bedrohlich, sie reiht sich aber ein in ständige Standort- und Arbeitsplatzbedrohungen, sodass eine gewisse Krisenroutine entsteht: „An dieses ständige Infragestellen vom Arbeitsplatz gewöhnt man sich eigentlich auch.“

(c) Wir sind aber auch im Zentrum der Krise auf eine ganz gegenteilige Wahrnehmung gestoßen: Die Krise erscheint als „machtpolitische Inszenierung“ derjenigen, die von ihren Folgen profitieren: „Die angebliche Krise wird vom Unternehmen hergenommen, um Profite zu erhöhen.“ - „Durch die ganzen Medien, unsere Banken, wird das alles so hochgespielt. Die haben die Krise. Die haben sie. Man sieht es ja. Die haben ja alle Miese.“

Schon dieser kurze Aufriss zeigt: Die Große Krise ist kein Ereignis, das die Verhältnisse für alle in ein neues Licht taucht und damit

1 Dies gilt nicht für die Studien aus Jena. Sie argumentieren allerdings auf einer recht spezifischen empirischen Basis, nämlich quantitativen Befragungen in einigen Großunternehmen.

2 Wir haben qualitative Erhebungen in zwei gewerkschaftlichen Bildungsstätten durchgeführt und dabei fünf Gruppendiskussionen (mit insgesamt 32 Teilnehmern) sowie 20 leitfadengestützte Einzelinterviews geführt. Die Interviewten kamen aus insgesamt 16 unterschiedlichen Betrieben. Das Sample ist selbstverständlich nicht repräsentativ. Befragte aus typischen gewerkschaftlichen Kernsektoren (Betriebe mit hohem Organisationsgrad, in der Automobilindustrie Betriebe mit funktionierender Vertrauensleutearbeit) sind überproportional vertreten, ebenso Männer sowie gewerblich Beschäftigte. In den empirisch basierten Hypothesen, die wir im Folgenden darstellen, drücken sich die Wahrnehmungen und Handlungsorientierungen einer spezifischen - gewerkschaftsaffinen Gruppe der Industriearbeiterschaft aus; sie sollten daher nicht vorschnell verallgemeinert werden. Die Studie wurde finanziell unterstützt von der Rosa-Luxemburg-Stiftung und der WolfgangAbendroth-Stiftungsgesellschaft.

3 Insgesamt haben wir in unserer Studie fünf Typen der Wahrnehmung der persönlichen Krisenbetroffenheit unterschieden, von denen wir hier die drei wichtigsten herausgreifen (Detje et al. 2011, S. 53ff.). Die Typisierung erfolgte subjektbezogen; es zeigt sich allerdings ein recht deutlicher Zusammenhang zwischen Betriebszugehörigkeit und wahrgenommener Krisenbetroffenheit.

4 Dieses Zitat wie auch die folgenden stammen aus unseren qualitativen Interviews und Gruppendiskussionen (vgl. Fußnote 2). 
die interessen- und handlungspolitischen Optionen neu sortiert. Unser Befund: Die Krise wird in ihrem bisherigen Verlauf nur von Minderheiten als biografischer Einschnitt erlebt, mehrheitlich hingegen als Bestätigung langjähriger Erfahrungen wahrgenommen. Ein direkter Rückschluss von „objektiver“ Krisenbetroffenheit auf die subjektive Krisenwahrnehmung ist dabei kaum möglich. Zum einen, weil sich gerade unter den „Krisenopfern“ viele finden, die vor dem Hintergrund einer durch Unsicherheit und Brüche geprägten Erwerbsbiografie eine generalisierte Kompetenz beim Umgang mit prekären Lebens- und Beschäftigungsverhältnissen entwickelt haben. Zum andern, weil gerade jene die Krise als „herben Schlag“ wahrnehmen, die bislang unter vergleichsweise gesicherten Bedingungen gearbeitet haben.

Entscheidend kommt ein dritter Befund hinzu: Die Krisenursachen sind nebulöser denn je. Das hat maßgeblich mit dem spezifischen Charakter dieser Krise zu tun. Kennzeichnend sind nicht in erster Linie realwirtschaftliche Überproduktion oder krisenhafter Strukturwandel, wie er beispielsweise in den Studien der 1970er und frühen 1980er Jahre zum Krisenbewusstsein im Mittelpunkt stand. Die damaligen, stärker branchenbezogenen Krisenprozesse - beispielsweise die „marktinduzierte Krise“ der Werftindustrie oder die „technikinduzierte Krise“ der Elektroindustrie (Zoll 1981, 1984; vgl. auch Schumann et al. 1982; Kubach et al. 1985) - hatten eine deutliche höhere „Handgreiflichkeit" als die jüngsten Krisenprozesse, die zwar als betrieblich durchaus folgenreich erlebt werden, ihre Auslöser aber in entfernten und komplexen Prozessen in den US-amerikanischen Immobilien- und Finanzmärkten hatten. Die Verselbstständigung der Finanzmärkte, die vertrackten Formen der dortigen Aneignung wachsender Teile des gesellschaftlichen Reichtums, die spekulativen Formen der Verwertung von Geldkapital - all das sind Krisenprozesse jenseits des realwirtschaftlichen Nahbereichs, in denen scheinbar aus Geld mehr Geld gemacht wird, ohne irgendwelche „Werte“ produziert zu haben. Diese spezifische Mystifikation des ökonomischen Lebensprozesses macht es schwer, generalisierte Erkenntnisse aus unterschiedlichen Krisenerfahrungen zu gewinnen und interessenspolitisch zu verarbeiten.

Ein Vergleich mit der methodisch ähnlich gelagerten Studie von Bergmann et al. (2002) - auch hier wurden die Erhebungen in gewerkschaftlichen Bildungsstätten durchgeführt - zeigt andererseits, dass die jüngste Wirtschaftskrise viel eher als ein definierbares Ereignis, als eine gesellschaftliche und ökonomische Zuspitzung wahrgenommen wurde; dies auch aufgrund des massiven medialen Krisendiskurses. Demgegenüber war „Krise“ in der Vorgängerstudie ein aus Beschäftigtensicht eher als diffus erlebter Veränderungsprozess - u. a. geprägt durch nachlassende Normierungskraft von Tarifverträgen, stagnierende oder sinkende Löhne, steigende Leistungsanforderungen, Beschäftigungsabbau usw. -, der die eigene Verhandlungsposition unterminiert, nicht aber eine als konkreter Einschnitt erlebte tief gehende Rezession mit einem relativ klaren zeitlichen Horizont.

\section{3 \\ Betriebliche Krisenbewäl- tigung hinter der Fassade des „deutschen Beschäfti- gungswunders"}

Die Krise wird für die Einzelnen im Betrieb konkret spürbar und erlebbar. Deren Folgen und Bearbeitungsformen können Ansatzpunkte für korporatistische Verabredungen, aber auch für Kritik und Auseinandersetzungen sein. Mit drei zentralen Strategien, die wir im Hinblick auf ihre Bewertung durch die Beschäftigten näher betrachten, haben die Unternehmen auf die betrieblichen Herausforderungen der Wirtschaftskrise reagiert: ${ }^{5}$

\subsection{EXTERNALISIERUNGSSTRATE- GIEN: BESCHÄFTIGUNGSPUFFER LEIHARBEIT}

Der Abbau von Leiharbeit ${ }^{6}$ war - neben anderen Formen des Beschäftigungsabbaus (Entlassungen, aber auch die Beendung befristeter Beschäftigungsverhältnisse und die Nicht-Übernahme von Auszubildenden) - in fast allen Herkunftsbetrieben unserer Befragten der Puffer, um die Beschäftigung krisenbedingt zu flexibilisieren. Vor der Krise reicht der Anteil von Leiharbeitern von einigen Prozent bis hin zu 10-20 \% der Belegschaft, in einem Fall waren es sogar $50 \%$. In der Krise wurde die Leiharbeit stark reduziert, häufig auf null heruntergefahren - und dies nicht nur in denjenigen Betrieben und Abteilungen, die unmittelbar vom Auftragsrückgang betroffen waren. Teilweise wurden „frei“ gewordene Arbeitsplätze dann von Stammbeschäftigten eingenommen.

Die meisten Beschäftigten deuten solche Maßnahmen als praktisch alternativlos. Sie zeigen zwar Empathie mit denjenigen, die ihren Job verlieren, akzeptieren die Entlassung der Leiharbeiter aber als Element betrieblicher Beschäftigungssicherung: „In der Belegschaft jetzt, ja, war man einfach nur froh, dass es einen selber nicht getroffen hat. ... Also, es wurde in der Belegschaft schon diskutiert, ja, aber ja klar, bevor dass es uns trifft...".

In der Krise verschärft sich die Segmentierung zur Spaltung zwischen externer und interner Beschäftigung - und wirkt konfliktdämpfend. Zwischenzeitlich hatte es einigen Forschungsergebnissen zufolge danach ausgesehen, als würden sich die Leiharbeitsverhältnisse stärker in die bestehenden Belegschaftsstrukturen integrieren, sodass zwischen Kern- und Randbelegschaft nicht mehr deutlich unterschieden werden kann. In diesem $\mathrm{Zu}$ sammenhang war von neuen Solidarisierungspotenzialen zwischen Stamm- und Leiharbeitskräften die Rede (Holst 2009). In der Krise brechen allerdings die alten Spaltungslinien wieder auf und vertiefen sich. Der arbeitsrechtliche Statusunterschied, der zwischen Leih- und Stammbeschäftigten auch in Zeiten wirtschaftlicher Prosperität immer bestehen geblieben war,

5 Der Gute-Arbeit-Index des DGB gibt einen guten Überblick über die Verbreitung der verschiedenen Krisenreaktionsformen. An erster Stelle stehen Maßnahmen der internen Arbeitszeitflexibilisierung. Ein systematischer Abbau von Über- bzw. Plusstunden fand in $68 \%$ der Betriebe der Metallindustrie und jeweils $61 \%$ der Betriebe in Maschinenbau/Elektrogeräteherstellung sowie Fahrzeugbau statt. Kurzarbeit wurde - bezogen auf die in unsere Erhebung einbezogenen Branchen - in mehr als $50 \%$ der Betriebe eingeführt. Leiharbeit wurde in $58 \%$ (Fahrzeugbau), $51 \%$ (Maschinenbau/Elektrogeräte) bzw. $48 \%$ (Metallindustrie) der Betrieb abgebaut. Von „kriseninduzierten Umstrukturierungen “ waren 38-44\% der Betriebe betroffen.

6 Waren im Jahr 2008 noch 733.000 Leiharbeiter in Deutschland beschäftigt, so waren es ein Jahr später nur noch 621.000. Besonders kräftig sank die Leiharbeitsquote in der Metall- und Elektroindustrie von 6,3 auf 3,4\% (Gesamtwirtschaft: von 2,7 auf 2,2\% (IG Metall 2010b).

7 Solidarischen Lösungen - etwa erweiterte Kurzarbeit beim Stellenerhalt für die Leiharbeiter - sind allerdings rechtliche Grenzen gesetzt. Gleichwohl: Solche Lösungen standen in den Betrieben nicht ernsthaft zur Diskussion. 
schlägt wieder um in eine „harte“ Differenz: zwischen integriert und exkludiert, zwischen „arbeitend“ und „arbeitslos“.

Diese Grenzlinien wurden auch von den betrieblichen Interessenvertretungen nicht überwunden. Die ,intern Prekären“ sowie die Auszubildenden haben in der Betriebsratspolitik eindeutig Vorrang vor den „extern Prekären“. Die Rolle der Leiharbeit als beschäftigungspolitischer Flexibilitätspuffer wird selten infrage gestellt. Von Solidarisierung mit prekär Beschäftigten zur Sicherung der Gesamtbeschäftigung wurde uns kaum berichtet.

Auch die schnelle Kündigung von Leiharbeitskräften - und Leiharbeit steht hier stellvertretend auch für andere Formen befristeter Beschäftigung - gehört zum „deutschen Beschäftigungswunder“. Ihre Befriedungsfunktion wirkt allerdings nur negativ: als Stillhaltenotwendigkeit unter ökonomischen Krisenbedingungen. Die erneute rasante Zunahme der Leiharbeit beim (vorläufigen) Ausklang der Krise über Vorkrisenniveaus hinaus (vgl. Abschnitt 3.3) dokumentiert den prekären Charakter des Umbaus des Beschäftigungssystems - und damit eine soziale Realität, die als „permanente Krise“ wahrgenommen wird.

\subsection{ARBEITSZEITEN: POTENZIERTE FLEXIBILITÄT}

Quantitativ größere Bedeutung als der Abbau von Beschäftigung hat die interne Flexibilisierung mittels Arbeitszeitverkürzung. Insgesamt umfassten die Maßnahmen ein Zeitvolumen, das 1,2 Mio. Vollzeitäquivalenten entspricht (IG Metall 2010b, S. 5). Im Mai 2009 befanden sich 980.000 Beschäftigte in der Metall- und Elektroindustrie in Kurzarbeit. In 14 unserer 16 Referenzbetriebe fand Kurzarbeit statt davon interessanterweise auch in einigen derjenigen Betriebe, die als vergleichsweise krisenresistent beschrieben wurden.

Im Detail ist die Praxis der Kurzarbeit unter den Beschäftigten und Interessenvertretern durchaus umstritten. Insbesondere Verteilungsfragen - etwa zwischen gewerblichen und Angestelltenbereichen - wurden Gegenstand der Kritik, ferner organisatorische Defizite (z.B. verzögerte oder falsche Entgeltzahlungen, chaotische Schichtpläne) sowie vermutete Mitnahmeeffekte der Unternehmen. Dennoch: Im Grundsatz wird die Kurzarbeit nicht infrage gestellt. Auch sie gilt - ähnlich wie der Abbau von Leiharbeit - als prinzipiell unausweichlich: „Na ja, das müssen wir halt. Die Krise ist da. ... Wir sind ja froh, dass es über Kurzarbeit und nicht über Entlassungen abgefedert wird. Also klar.“

Von noch größerer Bedeutung ist in den Herkunftsbetrieben unserer Befragten die Flexibilisierung über Arbeitszeitkonten. ${ }^{8}$ Nicht selten wurden die Kontenspielräume erweitert, um ein weiteres Absenken der Arbeitszeiten zu ermöglichen. Minus-Stände von 200-300 Stunden pro Beschäftigten waren die Regel, in Einzelfällen gingen die Minuszeiten bis auf 700 Stunden. Um die Relationen zu verdeutlichen: 300 Stunden entsprechen umgerechnet 8,5 Arbeitswochen. Das reale Ausmaß der Flexibilisierung liegt noch weit darüber, denn vor der Krise waren die Konten in der Regel prall gefüllt. Diese Flexibilisierung über Zeitkonten war ganz sicher die geräuschloseste Form der Krisenbewältigung. Sie erfolgte weitgehend einvernehmlich zwischen den Betriebsparteien und ließ sich rechtlich wie organisatorisch einfach umsetzen: „Das ist nicht die Welt. Aber man hat gleich dazu gesagt, ob das jetzt minus 200 sind oder minus 300 oder 500, das ist uns egal. Hauptsache Arbeitsplatz."

Erklärbar wird die „Geräuschlosigkeit“, mit der diese Form der Zeitflexibilisierung vonstatten ging, nur vor dem Hintergrund einer bereits langfristig eingeübten Fähigkeit der Beschäftigten zur flexiblen Abstimmung der eigenen Lebensverhältnisse und der Alltagsorganisation auf die betrieblichen Zyklen der Zeitbeanspruchung. Die Freizeitgestaltung und die familiären Bedürfnisse den Anforderungen von Ökonomie und Betrieb unterwerfen zu müssen, ist keine neue Erfahrung. Gleichwohl führt die Krise in eine neue Dimension von Flexibilitätsanforderungen.

Hier werden weitere Schattenseiten des „german miracle“ deutlich. Für die Unternehmen haben sich erweiterte Flexibilisierungsspielräume aufgetan, und die Perspektive einer kapazitätsorientiert „atmenden“ Fabrik ist ein weiteres großes Stück nähergerückt. Für die Beschäftigten hingegen werden die Zumutungen einer „Ultra-Flexibilisierung“" zu einer nur noch schwer kompensierbaren Belastung - gerade zum Zeitpunkt nach der Krise, denn die Anpassungsleistungen „nach oben“ stehen erst dann an, wenn die Minusstunden wieder abgearbeitet und die Zeitkonten aufgefüllt werden müssen. ${ }^{9}$

\subsection{RESTRUKTURIERUNG: GUTE ARBEIT BLEIBT VIRULENT}

Bislang wenig Aufmerksamkeit haben Maßnahmen interner Reorganisation gefunden. Dabei handelt es sich u.a. um Verfahren der Relokalisierung der Arbeitskräfte innerhalb von Abteilungen und über Abteilungen und Bereiche hinweg. Gerade Großunternehmen können die ungleichzeitige Krisenbetroffenheit verschiedener Bereiche nutzen, mittels flexibler Personaleinsatzpolitik für einen Querausgleich $\mathrm{zu}$ sorgen. In der Automobilindustrie wurden so Beschäftigte aus hochpreisigen Produktbereichen in die Kleinwagenproduktion versetzt, die dank Abwrackprämie boomte (und wo zuvor die Leiharbeiter entlassen wurden).

Allerdings haben gerade diese krisendämpfenden internen Restrukturierungsmaßnahmen in einigen Fällen zu einem ausgeprägten individuellen Krisenerleben geführt: „Und dann hast du plötzlich ... so eine Krise. ... da bist du wieder eigentlich zurückgeschossen worden. Da warst du wieder ganz unten am Fließband. ... diesen Verdauungsprozess, den musst du erst mal [hin-]kriegen und den verkraften. ... ich weiß nicht, wann ich wieder zu mir gekommen bin. ... Aber - wie gesagt - im Innersten hast du gedacht: Hauptsache, ich hab einen Job. Aber es war im Unterbewusstsein, das hast du nicht rausgeholt. Das hast du keinem gesagt.“

Der interessante Punkt: „Hauptsache Job“ verweist nicht auf ein neues instrumentelles Arbeitsverständnis. Ansprüche auf Autonomie und Gute Arbeit sind nicht suspendiert, können vielmehr gerade in der Krise besonders virulent werden. Dies ändert nichts am „logischen Vorrang“ der Arbeitsplatzsicherheit, macht aber deutlich, dass nicht nur Arbeitsplatz- und Ein-

8 Dies gilt nicht nur für unsere Untersuchungsfälle. Lehndorff (2010b) schätzt, dass der Wegfall von Überstunden und der Abbau von Zeitkonten insgesamt mehr als die Hälfte der Reduzierung des Arbeitszeitvolumens in Deutschland ausmachte; der Anteil der Kurzarbeit lag demgegenüber bei rund einem Drittel.

9 Um noch ein Beispiel zu nennen: In einem Unternehmen bestanden im Mai 2009 im Durchschnitt 250 Minusstunden, bis Ende des Jahres dann wieder im Durchschnitt plus 100 Stunden. Das heißt, dass die Beschäftigten in 24 Arbeitswochen 350 Stunden „aufholen“ mussten - für jeden Einzelnen sind das im Durchschnitt 15 Plusstunden in der Woche. 
kommenssicherheit wichtig sind, sondern die arbeitsinhaltlichen Bedingungen: der Statusverlust („wieder ganz unten“) und die Angst, auf „Jedermann-Arbeiten“ zurückgefallen zu sein, wieder zum grauen Heer der Massenarbeiter zu gehören: „Mit der Tätigkeit identifiziere ich mich. Ich lebe das aus, ich liebe diesen Job. Und du bist als Facharbeiter da ... Und dann gehst du plötzlich in so eine Produktionslinie rein ... Hier muss ich die Hand hochheben, wenn ich auf die Toilette gehen möchte, dass mich dann einer ablöst. Hier hab ich keine Zeit, mal zwischendurch einen Kaffee zu holen. Hier hab ich keine Zeit, in mein Butterbrot mal zu beißen - außerhalb der Pausenzeiten. ... damit kam ... man nicht klar." Allerdings führt auch dieses (im Rahmen unserer Vergleichsfälle ziemlich ausgeprägte) Krisenerleben nicht zu artikuliertem Protest oder kollektiv ausgetragenen Konflikten. Vielmehr findet sich auch hier wieder das Deutungsmuster von Unausweichlichkeit und Alternativlosigkeit: „Also Proteste - wäre auch gar nicht möglich, weil gegen was willst du da protestieren?"

Widerstand scheint zwecklos. Die Konsequenzen, die der zitierte Beschäftigte aus der Krise zieht, sind konsequenterweise individualistisch. Er möchte seinen eigenen beruflichen Weg noch nachdrücklicher verfolgen und sich noch besser qualifizieren (Angestellte erwischt die Krise nicht so stark, so sein Argument). Der Befragte revidiert also nicht seine berufsbiografischen Orientierungen (etwa: „Da hätte ich mir die Ausbildung sparen können, wenn ich am Ende doch am Fließband lande"), sondern er intensiviert sie noch. Gegen den drohenden Verlust an Fachlichkeit hilft nur noch mehr fachliche Qualifikation.

Für alle drei betrieblichen Strategiefelder der Krisenbewältigung - die Externalisierungsstrategien durch den Abbau der Leiharbeit, die beiden internen Flexibilisierungen durch Arbeitszeitpolitik sowie die Relokalisierung der Arbeitskräfte - gilt also: Zwar kommt es verschiedentlich zu deutlicher Kritik an den spezifischen Umsetzungsformen, im Grundsatz werden die Maßnahmen aber weitgehend von den Interessenvertretungen und Beschäftigten mitgetragen. Dies geschieht vor dem Hintergrund von Deutungsmustern der Alternativlosigkeit und Unausweichlichkeit.

Positiverfahrungen, die Ansatzpunkte für neue Handlungsstrategien bilden könnten, bleiben die Ausnahme. Beispiel Arbeitszeitpolitik: Wir haben nur in Einzelfällen Anzeichen dafür gefunden, dass von den Beschäftigten neue Zeitansprüche entwickelt werden, beispielsweise aufgrund des positiven Erlebens der hinzugewonnenen „privaten“ Zeitressourcen. Von innovativen betrieblichen Politikansätzen, wie einem betrieblichen „Einrast-Effekt“, dem Fixieren der einmal verkürzten Arbeitszeiten, wie Lehndorff (2010a, 2010b) ihn ins Spiel gebracht hat, ganz zu Schweigen. Eher scheint auf die krisenbedingte Arbeitszeitreduzierung wieder eine massive Extensivierung zu folgen. Ähnliches gilt hinsichtlich der externen Flexibilisierungsstrategien. Der Höchststand an Leiharbeit (722.000 im Juli 2008) wurde nach dem massiven Einbruch auf 507.000 (April 2009) bereits im Sommer 2010 wieder überschritten. Mit 754.000 Beschäftigten lag die Zahl im August 2010 bereits wieder oberhalb des Vorkrisenniveaus (BA 2008-2010) und soll Prognosen zufolge bald auf über 1 Mio. ansteigen.

Die postfordistischen Flexibilisierungsstrategien werden also in und nach der Krise noch einmal intensiviert. Die Krise funktioniert nicht als Innovator; vielmehr folgt aus ihr eine verschärfte Gangart auf dem bereits eingeschlagenen Entwicklungspfad.

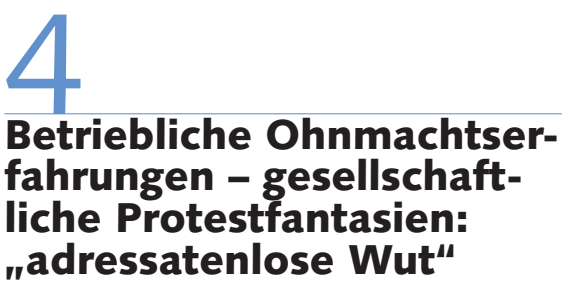

Dass die Beschäftigten in der akuten Krisensituation dem keinen Widerstand entgegensetzen, liegt keinesfalls an einer mangelnden Spürbarkeit von Krisenfolgen, sondern vielmehr an grundsätzlich fehlenden Perspektiven von Handlungsmöglichkeiten und Alternativen. Dies trifft zumindest für die betriebs- und unternehmenspolitische Ebene zu. Zwar werden hier die Krisenfolgen konkret „erlebt“, aber der Betrieb gilt der Mehrheit der Befragten nicht als relevanter Ort der Krisenbearbeitung: „Was willst du denn machen? Du kannst doch eh nichts machen. Die Spielregeln werden woanders ... also die Töne werden woanders erzeugt. Und wir haben bloß mit der Resonanz zu leben. Das ist einfach so.“
Ursache für Protest gibt es den Einschätzungen der Betroffenen nach in ausreichender Zahl, was fehlt, ist ein geeigneter Adressat - ist doch die Finanzmarkt- und die nachfolgende allgemeine Wirtschaftskrise nicht vor Ort im Betrieb verursacht. Zentral für viele Befragte ist ein verstärktes Erleben der eigenen Ohnmacht in der Krise. Zwar können manche Krisenfolgen, vor allem das Beschäftigungsrisiko, zumindest für die Kernbelegschaften in den Betrieben abgemildert werden - durchaus unter aktivem Zutun und mit Unterstützung der betrieblichen Interessenvertretung. Das Kernproblem wird dadurch freilich nicht berührt: einer entfernten, unbeherrschten finanzmarktkapitalistischen Dynamik ausgeliefert zu sein, wie sie ganz besonders in der Wirtschaftskrise konkret erlebbar wird. Erhebliches Protestpotenzial ist spürbar, es offenbart jedoch wenig Hoffnung und mehr Ratlosigkeit, wie die Verhältnisse zu ändern wären.

Das Ohnmachtserleben im Betrieb schlägt um in eine Art „adressatenlose Wut", die vom Betrieb auf die "Gesellschaft" und auf „Staat und Politik“ verschoben wird. Diese Wut schafft sich dort in relativ diffuser Weise Raum und führt zu Konflikterwartungen und zu ausgeprägten Protestfantasien, wie insbesondere unsere Gruppendiskussionen zeigen:

„Aber wenn das so weitergeht, dann werden sich die irgendwann mal alle organisieren und dann wird es wirklich mal krachen. Und ob das dann noch ruhig abgeht, das bezweifele ich. Weil da hat sich mittlerweile schon so viel angestaut, dass ich sagen muss, die werden wahrscheinlich bei irgendwas reagieren, was eigentlich gar nicht relevant ist ... Das steigert sich so langsam hoch, und dann kracht es, aber dann kracht es gewaltig, weil dann ich mich vielleicht sogar zu sagen traue, dass es vielleicht auch mit Gewalt was zu tun hat."

„Ja, da müssten andere Aktionen stattfinden wie eine Maikundgebung oder so was, da müssten in Deutschland die Straßen zu sein. Zu. Eine Woche lang. Da müsste sich nichts bewegen, gar nichts. Aber der Deutsche ist sowieso geduldig. Der wartet ab. Und wenn es scheppert, dann scheppert es richtig. Es wird immer wieder einen Neuanfang geben, ist meine Meinung. Irgendwann wird es wieder weitergehen. Aber wenn, dann muss richtig was passieren."

Brennende Reifen, „französische Verhältnisse" - immer wieder greifen die 
Befragten in unserer Erhebung auf solche Bilder zurück, mit denen sie ihrer Wut Ausdruck verschaffen; angedeutet werden dabei eher spontanistische Aktionen auf allgemein-gesellschaftlicher Ebene jenseits der Betriebe. Am ehesten richtet sich die artikulierte Wut - in recht abstrakter und kaum spezifizierter Weise, teilweise auch durchaus ressentimentgeladen - gegen „die Politiker“. Sie hätten sich von der Erfahrungswelt der Beschäftigten weitgehend abgekoppelt, seien aber zugleich selbst machtlos, so der Tenor der Gruppendiskussionen. Die Politik erscheint nicht als möglicher Problemlöser, sondern vielmehr selbst als Teil des Problems. Hoffnungen, dass ein entfesselter Finanzmarktkapitalismus durch politische Regulation erfolgreich eingehegt werden könnte, treten zurück hinter ein generalisiertes Misstrauen gegenüber Parteipolitikern und Mandatsträgern.

Allerdings gibt es in unserem Sample relevante Ausnahmen von der verbreiteten betrieblichen Konfliktlosigkeit (vgl. ausführlicher Detje et al. 2011). Dabei handelt es sich insbesondere um Betriebe mit einer bereits vor der Großen Krise bestehenden „Konfliktgeschichte“. In einigen Betrieben, die unter der ständigen Drohung einer Standortverlagerung stehen, haben Belegschaften und Interessenvertretungen gelernt, der ständigen Erpressung Widerstand entgegenzusetzen. Aus solchen Kämpfen ziehen die Beschäftigten die Erfahrung eigener Handlungsmacht: „Wir sagen: ,Bis hierhin und nicht weiter. Es reicht!' Es hat ja auch keinen Sinn mehr, was abzugeben." Diese erfahrene Handlungsmacht wird jedoch immer wieder begrenzt durch die strukturellen Abhängigkeiten (z.B. des Zulieferers vom fokalen Unternehmen). An der Abhängigkeit von den spezifischen ökonomischen Bedingungen und der strukturellen Krisenhaftigkeit ändern auch die partiellen Erfolge nichts. Es sind Abwehrkämpfe, keine offensiven Auseinandersetzungen auf der Grundlage eigener Alternativvorstellungen.

\section{Fazit}

Um die eingangs zitierten Hypothesen vor dem Hintergrund unserer Erhebungsergebnisse aufzugreifen: Der Augenschein einer „Krise ohne Konflikt“ ist durchaus nicht unzutreffend. Zwar sind großformatige gesellschaftliche Auseinandersetzungen hierzulande ausgeblieben. Und aus den Betrieben wird kaum von massiven Konflikten um die Krisenfolgen berichtet. Wie wir gesehen haben, gibt es dafür Gründe:

(1) Zunächst einmal wirkt die Krise nicht nur zwischen, sondern auch innerhalb der Betriebe eher differenzierend als dass sich Krisenerfahrungen vereinheitlichen würden. Schon allein daher sind die konkreten Ansatzpunkte kollektiven Handelns begrenzt.

(2) Für das Fehlen betrieblicher Auseinandersetzungen ist zentral, dass die Krisenbewältigung vor dem Hintergrund von tief sitzenden Vorstellungen von Alternativlosigkeit und fehlenden Handlungsmöglichkeiten stattfand. Zumal die Ursachen der Krisentendenzen nicht in den Betrieben lokalisiert werden, scheint hier ein geeigneter Adressat für Widerspruch und Protest zu fehlen.

(3) Und nicht zuletzt haben die arbeitsmarktpolitischen Erfolge der politischen und betrieblichen Krisenbewältigung konfliktdämpfend gewirkt. Indem das schwindende Arbeitsvolumen über eine flexibilisierte Zeitpolitik abgefedert wurde, wurden die Krisenfolgen eher zu einem Problem des individuellen Zeitmanagements als zu einem kollektiv geteilten Lohnarbeiterschicksal. Auch die Differenzierung zwischen Kern- und Randbelegschaften, die in der Krise wieder aufgebrochen ist, hat demobilisierende Funktionen, weil die Entlassung der Leiharbeiter die Stammbeschäftigten vor massiveren Krisenfolgen bewahrt hat.

Trotzdem bleibt hinter den Diagnosen einer „Krise ohne Konflikt“ verborgen, dass sich in den Betrieben ein hohes Potenzial an Angst und Wut aufgestaut hat. Letztere schafft sich eher in einem proklamierten abstrakten Willen zur Revolte denn in planvollem, zielgerichtetem politischem Handeln Ausdruck. Die teils vehement geäußerten politischen Protest- und Widerstandsvorstellungen sind zwar nicht unbedingt als konkrete, umsetzungsbezogene Handlungsorientierungen zu deuten. Gleichwohl sind sie mehr als bloßer praxisferner Verbalradikalismus. Sie können als Ausdruck von realen Ohnmachts- erfahrungen gedeutet werden, für die auf betrieblicher Ebene keine Artikulationsmöglichkeiten bestehen.

Auch die These vom „Krisenkorporatismus" ist nach dem Gesagten zu differenzieren, und zwar in zweierlei Hinsicht: Dass die betrieblichen Krisenbewältigungsmethoden relativ weitgehend von Beschäftigten und Interessenvertretungen mitgetragen werden, beinhaltet in unseren Untersuchungsfällen, erstens, keine vorbehaltlose Konsensorientierung gegenüber der Unternehmensseite. Die Befragten bleiben grundsätzlich auf kritischer Distanz; sie prüfen die Maßnahmen und bilden sich ein eigenes Urteil - das in den genannten Fällen gleichwohl so ausfällt, dass die durchgeführten Maßnahmen im Grundsatz unausweichlich sind. Aus diesem Grund halten wir auch die Thesen von Fatalismus und Apathie, von „Dumpfheit und Desorientierung“ (Offe 2010, S. 286) für zu kurz begriffen, denn wir sind nicht auf verfestigte Resignation, sondern eher auf kritischen Realismus gestoßen.

Zweitens, handelt es sich nicht um eine Rückkehr zum alten „Modell Deutschland“. Die beschäftigungspolitisch ausgesprochen erfolgreiche Krisenbewältigung in Deutschland basiert zwar einerseits auf einer weiterhin funktionierenden aktiven Arbeitsmarktpolitik des Staates (vor allem den Kurzarbeitsregelungen), andererseits fußt sie auf einem postfordistischen Unterbau, der durch Ultra-Flexibilisierung geprägt ist. Erst durch den vorangegangenen massiven Ausbau von Leiharbeit (externe Flexibilisierung) sowie durch die hochgradig flexiblen betrieblichen Arbeitszeitregime (interne Flexibilisierung) konnte es gelingen, dass die Krise hinsichtlich der Arbeitsplätze für die Kernbelegschaften relativ glimpflich verlief.

Das „german miracle“ ist aber auch mit erheblichen Lasten für die Beschäftigten verbunden: Die im europäischen Vergleich vergleichsweise hohe Arbeitsplatzsicherheit über die Krise hinweg ist erkauft mit einer noch einmal intensivierten Unterwerfung der Beschäftigten unter die rein ökonomisch definierten Determinanten der Zeitorganisation.

Zur relativen Konfliktarmut trägt, wie wir gesehen haben, außerdem bei, dass die Belegschaften häufig bereits ausgesprochen krisenerfahren und -gestählt sind - und auch dies entstammt nicht der Erfahrungswelt stabiler fordistischer Betriebsstrukturen und eines gelungenen konsensba- 
sierten deutschen Modells, sondern basiert vielmehr auf dem bereits tief sedimentierten Erleben von Prozessen „permanenter Reorganisation“, downsizing, der Verlagerung von Betriebsstätten usw.

Auch die Formel "guter Betrieb schlechte Gesellschaft" (Dörre et al. 2009, 2010) trifft unsere Forschungsergebnisse nur in Ausschnitten. Von einem „besonderen Firmenbewusstsein“, von Einschätzungen der Unternehmen als soziale Integrationsinstanz und Garanten sozialer Stabilität kann bei unseren Befragten bestenfalls in Einzelfällen (und zwar in einigen Großunternehmen) die Rede sein. Wenn der Betrieb integrierend wirkt, dann eher als eine „erzwungene Krisengemeinschaft", und dies nicht erst angesichts der "großen Krise“, sondern bereits im Erleben der jüngeren betrieblichen Geschichte als „permanente Krise“. Richtig ist diese For- mel in einem anderen Sinne: In der Tat werden als Schuldige der Krise und als Verantwortliche für deren Folgen kaum Akteure im eigenen Betrieb und Unternehmen ausgemacht. Zwar werden „Bänker" und „Finanzjongleure“ als Betreiber und schließlich auch wieder als Profiteure der Krise benannt. Und eine als von den Bedürfnissen und Erfahrungen der Beschäftigten abgekoppelt wahrgenommene Kaste der Politiker ist Adressat wachsender Kritik. Im Grundsatz wird die Wirtschaftskrise aber systemisch gedeutet. Die formulierte Kritik ist damit immer auch Systemkritik.

Die Krise wird gleichsam als Brennglas für lange Erfahrungen der Verschlechterung der Arbeits- und Lebensverhältnisse wahrgenommen. Das Systemversprechen einer vermeintlich sozialen Marktwirtschaft, dass hohe Leistungsbereitschaft mit
Anerkennung, Fortkommen und Sicherheit honoriert wird, ist in dem Erfahrungshorizont einer „permanenten Krise“ gesprengt. Wir haben es mit einem hohen Maß an Delegitimierung eines ökonomischen Systems zu tun, das Wohlfahrtsversprechen nicht einlöst - im Übrigen eine Entwicklung, auf die die Demoskopie bereits seit einiger Zeit verweist (Allensbach 2009; vgl. auch Bischoff et al. 2010, S. 102ff.).

Gegen die Systemkritik steht die Erfahrung alltäglicher Systemzwänge. Das erzeugt Ohnmacht (nicht Fatalismus) und Wut. Aber es bleibt die Hoffnung, den durch Systemlogik zementierten Status quo aufbrechen, neuen Gedanken und Veränderungsfantasien Raum verschaffen zu können. In diesem Dreieck zwischen - von systemischer Delegitimierung unterlegter - Ohnmacht, Wut, aber auch Hoffnung steckt die Zukunft.

\section{LITERATUR}

Allensbach (Institut für Demoskopie Allensbach) (2009): Die Berliner Republik. Allensbacher Jahrbuch für Demoskopie 2003-2009, Berlin/ New York

Bergmann, J./Bürckmann, E./Dabrowski, H. (2002): Krisen und Krisenerfahrungen. Einschätzungen und Deutungen von Betriebsräten und Vertrauensleuten, Supplement der Zeitschrift Sozialismus (4), Hamburg

Bischoff, J./Detje, R./Lieber, C./Müller, B./Siebecke, G. (2010): Die Große Krise. Finanzmarktcrash - verfestigte Unterklasse - Alltagsbewusstsein - Solidarische Ökonomie, Hamburg

Bundesagentur für Arbeit (BA) (2008-2010): Arbeitsmarkt in Zahlen Beschäftigungsstatistik, verschiedene Ausgaben, Nürnberg

Detje, R./Menz, W./Nies, S./Sauer, D. (2011): Krise ohne Konflikt? Interessen- und Handlungsorientierungen im Betrieb - die Sicht der Betroffenen, Hamburg

DGB-Index Gute Arbeit (2010): Der Report 2010. Wie die Beschäftigten die Arbeitsbedingungen in Deutschland beurteilen. Mit dem Schwerpunkt: Folgen der Finanzkrise, Arbeitsintensivierung, Berlin Dörre, K./Behr, M./Eversberg, D./Schierhorn, K. (2009): Krise ohne Krisenbewusstsein? Zur subjektiven Dimension kapitalistischer Landnahme, in: Prokla 39 (4), S. 559-579

Dörre, K./Hähnel, A./Holst, H./Matuschek, I. (2010): Guter Betrieb, schlechte Gesellschaft? Arbeits- und Gesellschaftsbewusstsein im Prozess kapitalistischer Landnahme, Jena, Ms.

Eichengreen, B./O'Rourke, K.H. (2010): A Tale of two depressions, www.voxeu.org

Heitmeyer, W. (Hrsg.) (2010a): Deutsche Zustände. Folge 8, Frankfurt a. $M$.

Heitmeyer, W. (2010b): Deutsche Zustände - Unruhige Zeiten. Presseinformation zur Präsentation der Langzeituntersuchung Gruppenbezogene Menschenfeindlichkeit am 3.12. in Berlin
Heitmeyer, W. (Hrsg.) (2010c): Deutsche Zustände. Folge 9, Frankfurt a. $M$.

Holst, H. (2009): Disziplinierung durch Leiharbeit? Neue Nutzungsstrategien von Leiharbeit und ihre arbeitspolitischen Folgen, in: WSI-Mitteilungen 62 (3), S. 143-149

IG Metall Vorstand Grundsatzfragen und Gesellschaftspolitik (2010a): Wirtschaft aktuell 2/2010 - aktuelle wirtschaftspolitische Analysen der IG Metall: Metallkonjunktur im Februar 2010, Frankfurt a. M.

IG Metall Vorstand Wirtschaft, Technologie, Umwelt (2010b):

Wirtschaftspolitische Informationen (5) vom 18. August, Frankfurt a. M. Köcher, R. (2009): Der Statusfatalismus der Unterschicht, in: Frankfurter Allgemeine Zeitung (FAZ) vom 16.12.

Krugman, P. (2009): Die neue Weltwirtschaftskrise, Frankfurt a. M./ New York

Kubach, N./Mautz, R./Schumann, M./Wittemann, K. P. (1985): Krisenpolitik und Belegschaftsverhalten. Metallarbeiter zwischen Gegenwehr und Unterwerfung, Hamburg

Lehndorff, S. (2010a): Arbeitszeitpolitik nach der Kurzarbeit, in: Schwitzer, H./Ohl, K./Rohnert, R./Wagner, H. (Hrsg.): Zeit, dass wir das drehen! Perspektiven der Arbeitszeit- und Leistungspolitik, Hamburg, S. 39-62

Lehndorff, S. (2010b): Chancen der Arbeitszeitverkürzung, in: Gegenblende (05), September/Oktober, www.gegenblende.de Menz, W./Detje, R./Nies, S./Sauer, D./Keller, N. (2011): Zeit- und Leistungspolitik in und nach der Wirtschaftskrise - die Perspektive der Betroffenen, in: Schröder, L./Urban, H.-J. (Hrsg.): Gute Arbeit. Folgen der Krise, Arbeitsintensivierung, Restrukturierung, Köln, S. 99-113 Offe, C. (2010): "Keine Aussicht auf eine Repolitisierung in Zeiten der Krise“. Claus Offe im Gespräch mit Gunter Hofmann und Wilhelm Heitmeyer, in: Heitmeyer, W. (Hrsg.): Deutsche Zustände. Folge 8, Berlin, S. $283-295$ 
Pickshaus, K./Urban, H.-J. (2011): Das Nach-Krisen-Szenario: Beschäftigungspolitische Entspannung und arbeitspolitische Problemzuspitzung?, in: Schröder, L./Urban, H.-J. (Hrsg.): Gute Arbeit 2011. Handlungsfelder für Betriebe, Politik und Gewerkschaften, Frankfurt a. M. Schumann, M./Einemann, E./Siebel-Rebell, C./Wittemann, K. P.

(1982): Rationalisierung, Krise, Arbeiter. Eine empirische Untersuchung der Industrialisierung auf der Werft, Frankfurt a. M.
Zoll, R. (Hrsg.) (1981): Arbeiterbewusstsein in der Wirtschaftskrise. Erster Bericht: Krisenbetroffenheit und Krisenwahrnehmung, Köln Zoll, R. (Hrsg.) (1984): „Die Arbeitslosen, die könnt' ich alle erschieBen". Zweiter Bericht: Arbeiterbewusstsein in der Wirtschaftskrise, Köln 